\section{International Scientific Journal Theoretical \& Applied Science}

\author{
p-ISSN: 2308-4944 (print) e-ISSN: 2409-0085 (online) \\ $\begin{array}{lll}\text { Year: } 2016 \quad \text { Issue: } 7 & \text { Volume: } 39\end{array}$ \\ Published: $30.07 .2016 \quad$ http://T-Science.org
}

\begin{abstract}
Master of Engineering and Technology, Corresponding
Member of International Academy of Theoretical and Applied Sciences, Lecturer of Vladimir Industrial College, Russian Federation chemezov-da@yandex.ru
\end{abstract}

SECTION 7. Mechanics and machine construction.

\title{
THE DRAWING OF THE PLATE STOCK WITHOUT BLANK HOLDER
}

Abstract: The article is presented the character of plastic deformation of the workpiece material during performing of the forming operation of the sheet-metal stamping. The results of calculation of stress-strain state of the material in the conditions of the wrinkles formation on the flange of the deformable plate stock are considered.

Key words: plate stock, flange, wrinkles formation, deformation, energy.

Language: Russian

Citation: Chemezov DA (2016) THE DRAWING OF THE PLATE STOCK WITHOUT BLANK HOLDER. ISJ Theoretical \& Applied Science, 07 (39): 1-6.

Soi: http://s-o-i.org/1.1/TAS-07-39-1 Doi: crossef http://dx.doi.org/10.15863/TAS.2016.07.39.1

\section{ВЫТЯЖКА ЛИСТОВОЙ ЗАГОТОВКИ БЕЗ СКЛАДКОДЕРЖАТЕЛЯ}

Аннотация: В статье представлен характер пластической деформации материала заготовки при выполнении формообразующей операции листовой итамповки. Рассмотрень результать расчета напряженно-деформированного состояния материала в условиях образования складок на фланце деформируемой листовой заготовки.

Ключевые слова: листовая заготовка, фланец, образование складок, деформация, энергия.

\section{Введение}

Образование складок (гофрообразование) на фланце листовой заготовки в процессе вытяжки является дефектом, приводящим к ухудшению качества обработанных поверхностей и к отклонению от требуемой формы полой тонкостенной детали $[1,2,3,4,5]$. Интенсивность образования складок на не прижатом фланце возрастает с увеличением наружного диаметра и уменьшением толщины листовой заготовки. Малый зазор между контактными поверхностями пуансона и матрицы позволяет сгладить складки на поверхностях продавливаемой листовой заготовки. Этот процесс приводит к увеличению усилия, которое прикладывается к пуансону для реализации вытяжки тонкостенной детали. Визуальное представление характера деформации материала и расчет энергетических и силовых факторов процесса неглубокой вытяжки заготовки без складкодержателя можно получить методом твердотельного моделирования листовой штамповки в специальных компьютерных программах.

\section{Материалы и методы исследования}

Процесс неглубокой вытяжки стальной листовой заготовки типа «диск» осуществлялся в модулях Explicit Dynamics и Autodyn программной среды Ansys.

Модели пуансона (part 1), листовой заготовки (part 2) и матрицы (part 3) были сопряжены между собой для выполнения процесса вытяжки.

Обработке давлением подвергалась модель листовой заготовки, имеющая физикомеханические свойства конструкционной стали. Для листовой заготовки было принято уравнение состояния материала Linear (линейное) co следующими свойствами: исходная плотность 7.85 г/см ${ }^{3}$, модуль объемной упругости - 1.666667 $\times 10^{8}$ кПа, исходная температура - 295.149994 К, удельная теплоемкость - 434 Дж/(кг × К). Модель прочности материала - Elastic (упругая) при модуле сдвига равным $7.692308 \times 10^{7}$ кПа. Минимальный коэффициент плотности (SPH) материала принят величиной 0.2 , максимальный коэффициент плотности материала - 3.0.

Контактное взаимодействие моделей формообразующих инструментов и листовой заготовки (тип контакта Lagrange/Lagrange) 
осуществлялось по алгоритму траектории контакта. Этот алгоритм может применяться для всех 3D неструктурированных решателей. Принятый алгоритм контакта имеет следующие преимущества по сравнению с другими алгоритмами контактов [6]:

1. Нет особых требований для задания зоны контакта и возможность создания физического зазора между поверхностями моделей в начале моделирования.

2. Нет ограничений по времени контактного взаимодействия моделей. При снятии ограничений можно уменьшить время процесса расчета.

3. Возможен расчет энергетических параметров моделируемого процесса.

Принимались условия контакта Penalty, при которых равные и противоположные силы рассчитываются на узлах модели в целях сохранения линейного и углового моментов. Для удовлетворения условия контакта требуется несколько циклов расчета с сохранением инерции вырожденных узлов.

Усилие, действующее на вытяжной пуансон, принималось величиной 50 кН.

Контроль решения производился по следующим параметрам [7]: линейная вязкость 0.2, метод Lagrange/ALE с обновленной плотностью - автоматический, метод Эйлера для расчета скорости деформации средневзвешенный, метод Эйлера для расчета давления - средний, ALE/Euler энергия - полная, коэффициент деформации вырождения элементов (узлов) модели заготовки - 1.5.

Схема процесса неглубокой вытяжки листовой заготовки без складкодержателя и размеры твердотельных моделей представлены на рис. 1.

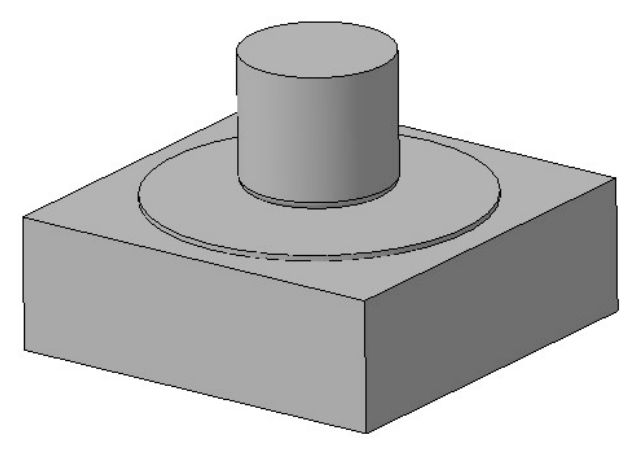

\begin{tabular}{c}
\hline Размеры заготовки \\
\hline Наружный диаметр -128 мм \\
Толщина -2 мм \\
Размеры пуансона \\
\hline Наружный диаметр -57 мм \\
Длина -50 мм \\
Радиусная фаска на рабочей части -3 мм \\
Размеры матрицы \\
Длина $\times$ Ширина $\times$ Высота $-150 \times 150 \times 50$ мм \\
Диаметр формообразующего отверстия -64 мм \\
Радиусная фаска в отверстии -5 мм
\end{tabular}

Рисунок 1 - Схема процесса неглубокой вытяжки листовой заготовки и размеры моделей.

Результаты и их обсуждение

На рис. 2 представлен характер деформации материала после вытяжки листовой заготовки на глубину 10 мм при времени выполнения процесса 0.5 мс.

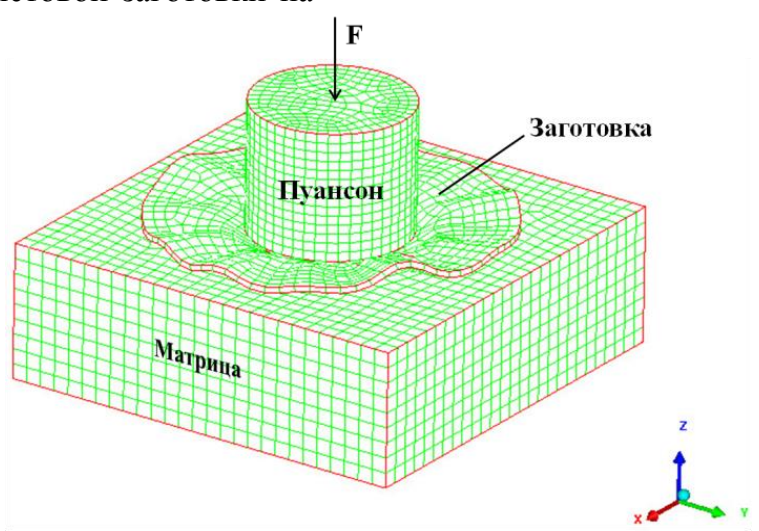

Рисунок 2 - Деформированное состояние модели листовой заготовки.

Величины полной деформации материала (а), напряжения (интенсивность) в материале (б), полной скорости деформации материала (в), напряжения по фон Мизесу в материале (г), давления на поверхности листовой заготовки (д), давления на твердое тело, обладающее вязкими свойствами (е), внутренней энергии деформации материала (ж), изменения объема материала (з) и абсолютной скорости движения материала (и) представлены контурами на модели листовой заготовки (рис. 3). 


\begin{tabular}{|c|c|c|c|c|c|c|}
\hline Impact Factor: & $\begin{array}{l}\text { ISRA (India) } \\
\text { ISI (Dubai, UAE } \\
\text { GIF (Australia) } \\
\text { JIF }\end{array}$ & $\begin{array}{l}=1.344 \\
=0.829 \\
=0.564 \\
=1.500\end{array}$ & $\begin{array}{l}\text { SIS (USA) } \\
\text { PИНЦ (Russia) } \\
\text { ESJI (KZ) } \\
\text { SJIF (Morocco) }\end{array}$ & $\begin{aligned}= & 0.912 \\
= & 0.234 \\
= & 1.042 \\
= & \mathbf{2 . 0 3 1}\end{aligned}$ & $\begin{array}{l}\text { ICV (Poland) } \\
\text { PIF (India) } \\
\text { IBI (India) }\end{array}$ & $\begin{array}{l}=6.630 \\
=1.940 \\
=4.260\end{array}$ \\
\hline
\end{tabular}
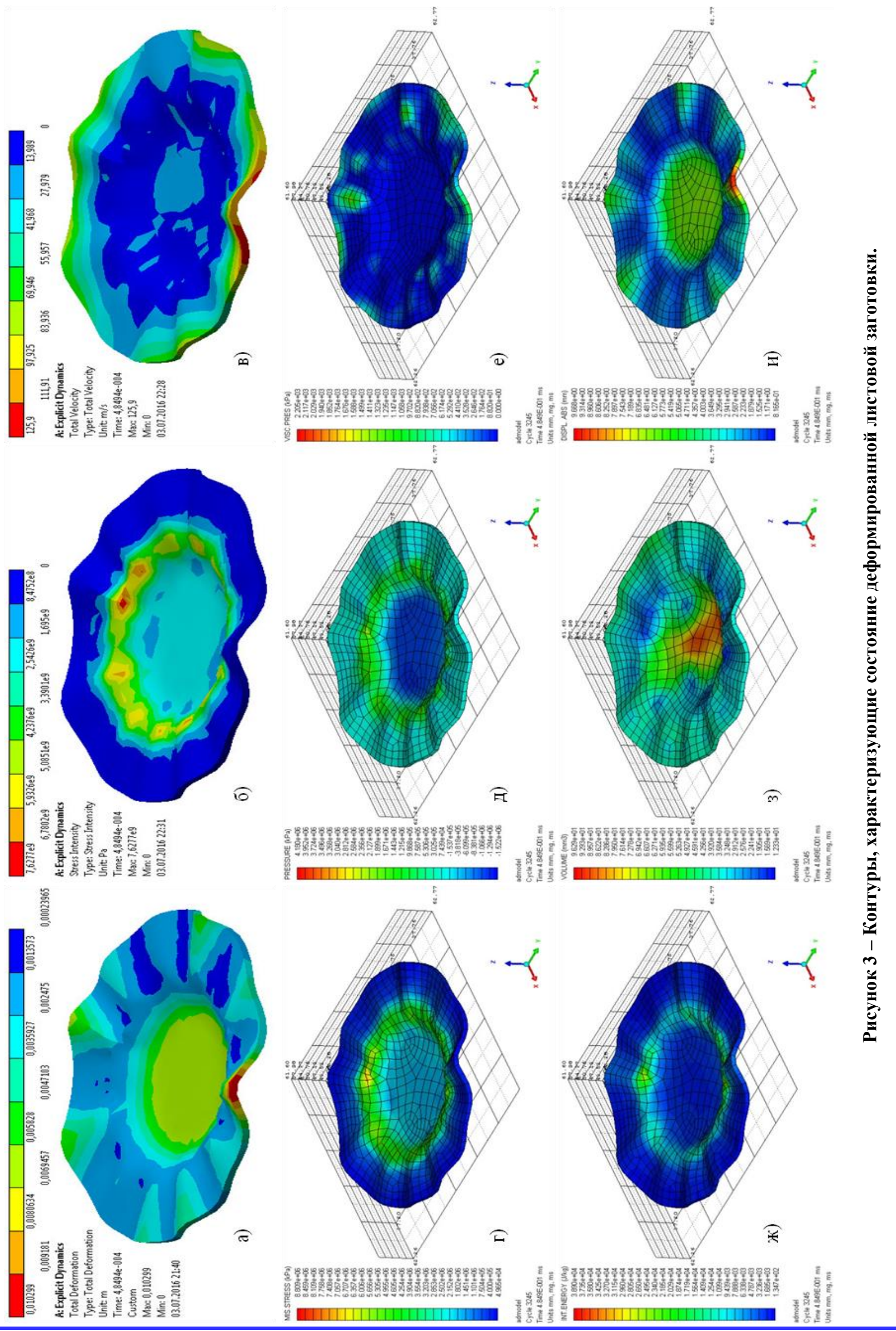

ISPC Results and Perspectives, Harrisburg, USA 


\begin{tabular}{|c|c|c|c|c|c|c|}
\hline Impact Factor: & $\begin{array}{l}\text { ISRA (India) } \\
\text { ISI (Dubai, UAF } \\
\text { GIF (Australia) } \\
\text { JIF }\end{array}$ & $\begin{array}{l}=1.344 \\
=0.829 \\
=0.564 \\
=1.500\end{array}$ & $\begin{array}{l}\text { SIS (USA) } \\
\text { PИНЦ (Russia) } \\
\text { ESJI (KZ) } \\
\text { SJIF (Morocco) }\end{array}$ & $\begin{array}{l}=0.912 \\
=0.234 \\
=1.042 \\
=2.031\end{array}$ & $\begin{array}{l}\text { ICV (Poland) } \\
\text { PIF (India) } \\
\text { IBI (India) }\end{array}$ & $\begin{array}{l}=6.630 \\
=1.940 \\
=4.260\end{array}$ \\
\hline
\end{tabular}

Графики зависимости энергии деформации материала и величины механического движения заготовки [8] по трем координатным осям от

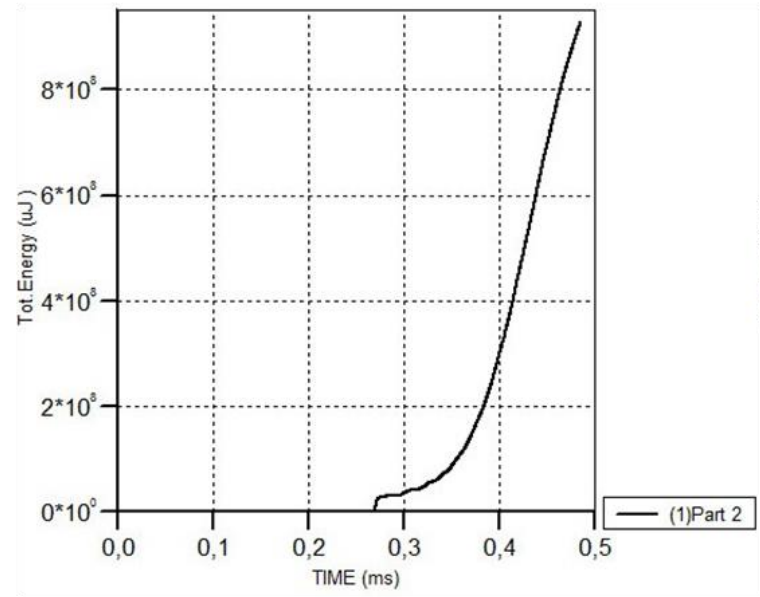

a)

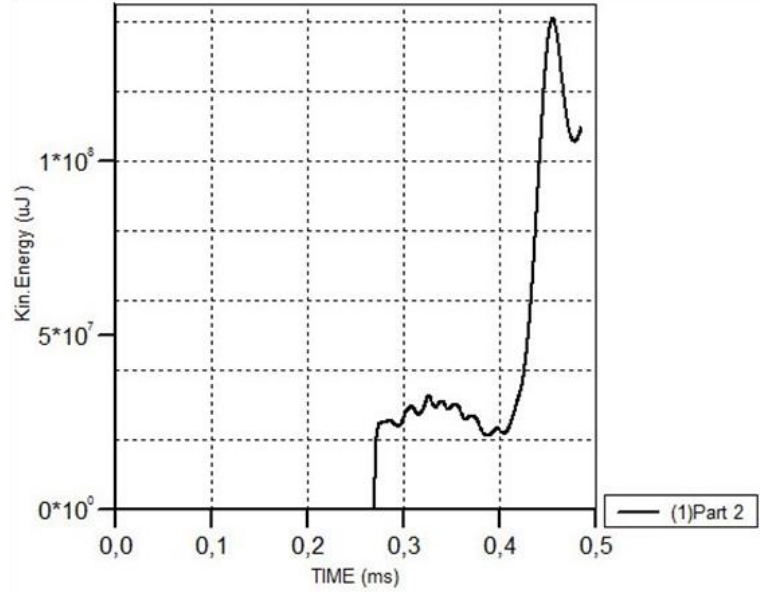

B)

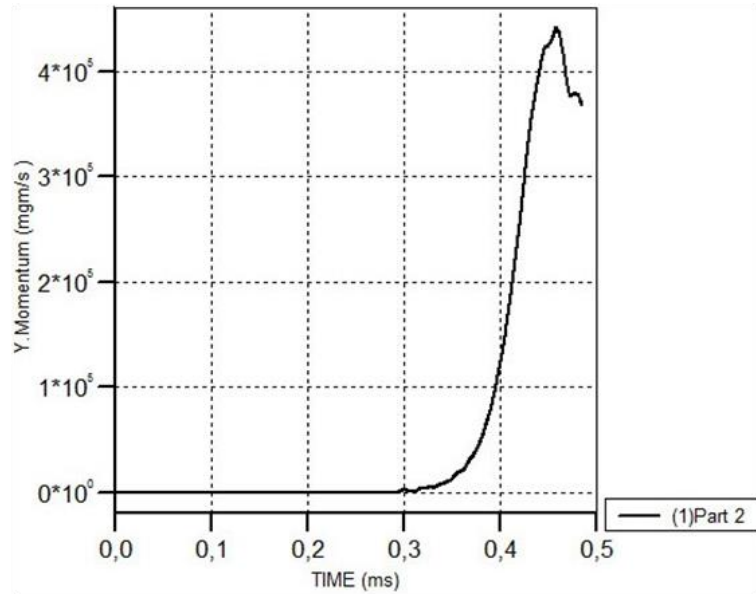

д) времени процесса вытяжки представлены на рис. 4.

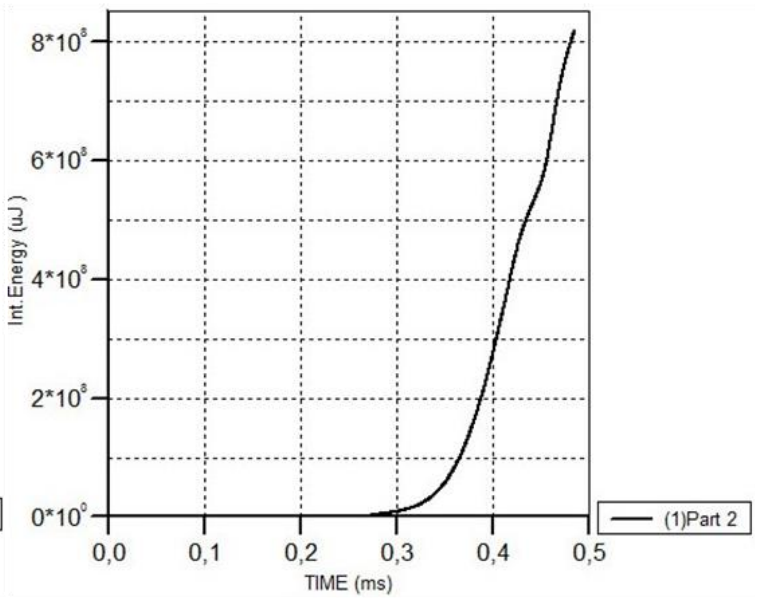

б)

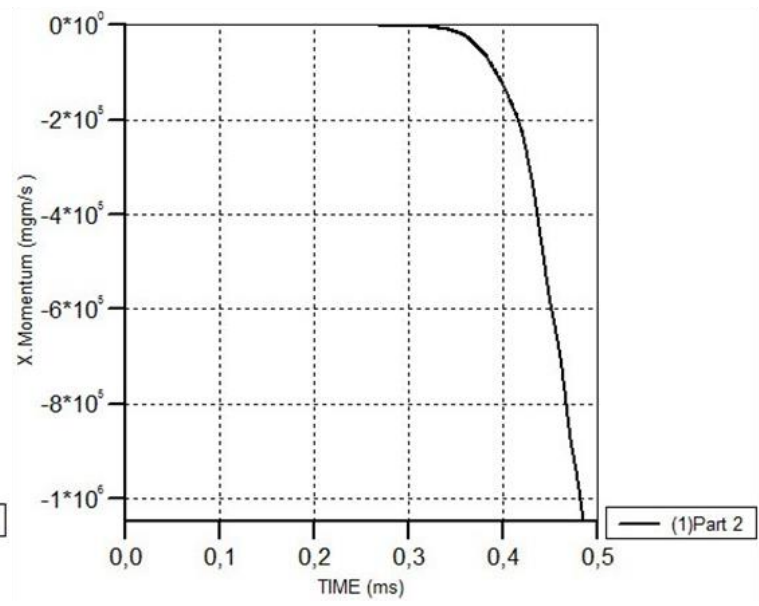

ए)

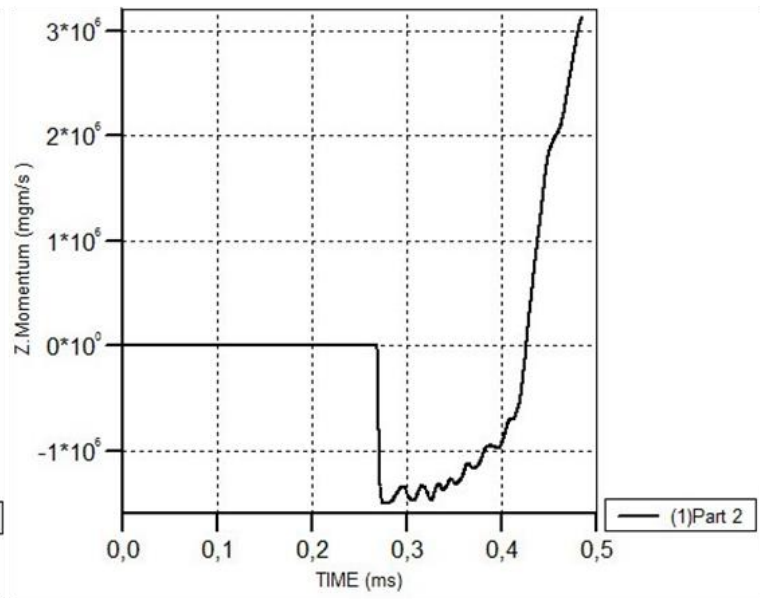

e)

Рисунок 4 - Зависимости: а - полной энергии деформации материала заготовки от времени процесса вытяжки, б - внутренней энергии деформации материала заготовки от времени процесса вытяжки, в - кинетической энергии деформации материала заготовки от времени процесса вытяжки, г механического движения листовой заготовки (импульс) по оси Х от времени процесса вытяжки, Д механического движения листовой заготовки (импульс) по оси Ү от времени процесса вытяжки, е механического движения листовой заготовки (импульс) по оси $\mathrm{Z}$ от времени процесса вытяжки. 


\begin{tabular}{|c|c|c|c|c|c|c|}
\hline Impact Factor: & $\begin{array}{l}\text { ISRA (India) } \\
\text { ISI (Dubai, UAF } \\
\text { GIF (Australia) } \\
\text { JIF }\end{array}$ & $\begin{array}{l}=1.344 \\
=0.829 \\
=0.564 \\
=1.500\end{array}$ & $\begin{array}{l}\text { SIS (USA) } \\
\text { PИНЦ (Russia) } \\
\text { ESJI (KZ) } \\
\text { SJIF (Morocco) }\end{array}$ & $\begin{array}{l}=0.912 \\
=0.234 \\
=1.042 \\
=2.031\end{array}$ & $\begin{array}{l}\text { ICV (Poland) } \\
\text { PIF (India) } \\
\text { IBI (India) }\end{array}$ & $\begin{array}{l}=6.630 \\
=1.940 \\
=4.260\end{array}$ \\
\hline
\end{tabular}

Ввиду малой жесткости «диска» и избыточного материала на фланцевой части начинают образовываться складки от усилия, приложенного к центральной части листовой заготовки.

Дадим общую оценку состояния материала листовой заготовки после вытяжки еe на заданную глубину. Наибольшей деформации подвергаются дно формирующейся полой тонкостенной детали и фланец листовой заготовки. Разные размеры складок приводят к неравномерной деформации фланца листовой заготовки. При этом максимальное напряжение возникает в области изгиба (радиусная фаска в отверстии матрицы) материала листовой заготовки $[9,10,11]$. Степень изменения формы фланца деформируемой листовой заготовки характеризуется растяжением или сжатием участков материала. Давление на материал листовой заготовки увеличивается в $10-11$ раз. Наибольшая внутренняя энергия (нагревание) деформации материала приходится на изгиб заготовки в области радиусной фаски в отверстии вытяжной матрицы. Объем материала при деформации листовой заготовки изменяется на различных участках от 46 мм $^{3}$ (фланец) до 86 мм $^{3}$ (дно формируемой тонкостенной детали). Отмечено значительное перемещение материала в зоне наибольших по величине образовавшихся складок на фланце листовой заготовки и на дне формируемой тонкостенной детали.

Кинетическая энергия возникает при движении (перемещении) материала листовой заготовки. Материал начинает неравномерно нагреваться (взаимодействие частиц), и тем самым возникает внутренняя энергия. Совокупность кинетической, внутренней и других энергий, возникающих при деформации материала листовой заготовки, составляют полную энергию. Полная и внутренняя энергии по величине и характеру изменения на всем временном диапазоне процесса вытяжки листовой заготовки практически одинаковы. Кинетическая энергия деформации материала в 6 - 7 раз меньше, чем внутренняя (полная) энергия. На графике (рис. 4, в) отмечены следующие изменения кинетической энергии: на временном диапазоне 0.27 - 0.4 мс - сопротивление материала при воздействии на него рабочей части пуансона, на временном диапазоне $0.4-0.45$ мс изгиб заготовки и последующее продавливание материала в рабочее отверстие матрицы (резкое увеличение энергии).

Импульс может иметь как положительные, так и отрицательные значения в зависимости от направления движения листовой заготовки. При сравнении величин импульсов по осям $\mathrm{X}$ и $\mathrm{Y}$ глобальной системы координат (рис. 2) можно отметить, что листовая заготовка движется в разных направлениях. Импульс заготовки по координатной оси $\mathrm{X}$ в 2 раза больше, чем импульс заготовки по оси Ү. По координатной оси Z наблюдается переменное движение материала листовой заготовки. Это связано с упругими деформациями материала в момент приложения нагрузки рабочей частью вытяжного пуансона (отрицательные значения) и последующими пластическими деформациями материала листовой заготовки (положительные значения). Импульс заготовки по координатной оси $\mathrm{Z}$ в 3 раза больше, чем импульс заготовки по оси X (часть механического движения вытяжного пуансона переходит в движение заготовки).

Единицы измерения на графиках: для энергий - микроджоуль (мкДж), для импульса мг $\times$ м/c, для времени - миллисекунда (мс).

\section{Заключение}

В соответствии с выполненным анализом технологического процесса вытяжки стальной листовой заготовки можно сделать следующие выводы:

1. Образование складок на фланце приводит к увеличению затрат мощности оборудования для осуществления обработки материала давлением.

2. Применение в вытяжном штампе складкодержателя, прижимаемого с усилием к фланцу листовой заготовки, полностью не устраняет образование складок, а способствует значительному уменьшению их размеров, что приводит к сбалансированности процесса вытяжки и равномерной деформации материала.

\section{References:}

1. Chemezov DA, Seliverstov VS, Kondrakov AA (2015) The process of corrugation on the flange of the deformable of the plate stock. Journal scientific and applied research, №10/2015. pp. 79 - 81. ISSN 2306-9147.
2. Chemezov DA, Seliverstov VS (2015) The intensity of the formation of corrugation on the flange of the deformable plate stock of thickness $1-5 \mathrm{~mm}$. Scientific and theoretical 


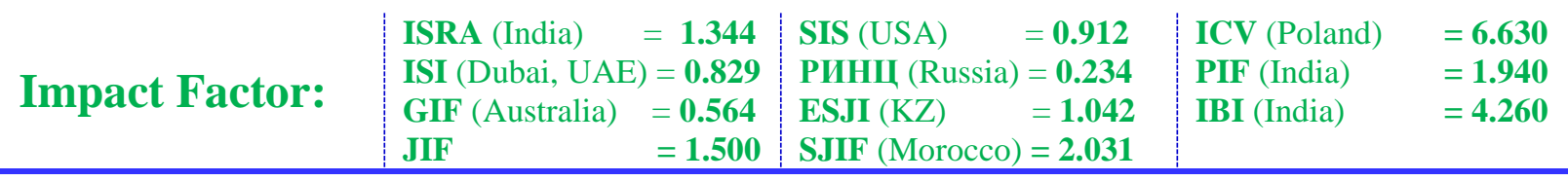

journal «Systems Engineering», №2. - pp. 71 76. ISSN 2304-0823.

3. Chemezov DA (2015) The research of the shallow drawing process of the plate stock. ISJ Theoretical \& Applied Science 10 (30): 11-15. Soi: http://s-o-i.org/1.1/TAS-10-30-4 Doi: http://dx.doi.org/10.15863/TAS.2015.10.30.4

4. Yakovlev SS, Remnev KS, Kalashnikov AE (2011) The influence of mechanical properties anisotropy on the buckling in axisymmetric details drawing process. Proceedings of the TSU, № 4/2011. - pp. 98 - 108.

5. Yakovlev SS, Remnev KS (2014) Wrinkling of axisymmetric parts made by drawing of an anisotropic material. Proceedings of Higher Educational Institutions. Machine Building, № $9(654) / 2014$. - pp. 39 - 47.

6. (2016) Interactions - Lagrange/Lagrange Introduction. Available: https://www.sharcnet.ca/Software/Ansys/16.2.3 lenus/help/wb_adyn/adyn_inter_laglag_intro.html (Accessed: 10.07.2016).

7. Autodyn. Explicit Software for Nonlinear Dynamics. User Manual. Version 6.1. 2005, Century Dynamics, Inc.
8. (2016)

Impuls.

Available: https://ru.wikipedia.org/wiki/Импульс

(Accessed: 10.07.2016).

9. Chemezov DA, Seliverstov VS, Komisar AS, Zezina NA, Tyurina SI (2015) Stamping of the plate stock with blank holder: the character of the material deformation and calculation of the coefficient of elongation. ISJ Theoretical \& Applied Science 11 (31): 101-107. Soi: http://so-i.org/1.1/TAS-11-31-16 Doi: http://dx.doi.org/10.15863/TAS.2015.11.31.16

10. Chemezov DA, Seliverstov VS, Bayakina AV, Zezina NA (2016) The influence of the magnitude of the radius chamfer in the die hole on the degree of deformation of the processed material and the productivity of the deep drawing process of the plate stock. ISJ Theoretical \& Applied Science, 01 (33): 52-57. Soi: http://s-o-i.org/1.1/TAS-01-33-11 Doi: http://dx.doi.org/10.15863/TAS.2016.01.33.11

11. Chemezov DA (2016) The calculation of the maximum stress of thin-walled detail while performing the technological process of deep drawing of the plate stock. XIII International scientific-practical conference "Fundamental and applied researches in the modern world". Vol. 1. - pp. 36 - 39. ISSN 2307-1354. 